\title{
ON THE UNIQUENESS OF MAXIMAL OPERATORS FOR ERGODIC FLOWS
}

\author{
Lasha EPHREMIDZE
}

\begin{abstract}
The uniqueness theorem for the ergodic maximal operator is proved in the continuous case.
\end{abstract}

Let $(X, \mathbb{S}, \mu)$ be a finite measure space,

$$
\mu(X)<\infty
$$

and let $\left(T_{t}\right)_{t \geq 0}$ be an ergodic semigroup of measure-preserving transformations of $(X, \mathbb{S}, \mu)$. As usual the map $(x, t) \rightarrow T_{t} x$ is assumed to be jointly measurable. For an integrable function $f, f \in L(X)$, the ergodic maximal function $f^{*}$ is defined by equation

$$
f^{*}(x)=\sup _{t>0} \frac{1}{t} \int_{0}^{t} f\left(T_{\tau} x\right) d \tau, \quad x \in X .
$$

We claim that the following uniqueness theorem is valid for the maximal operator $f \rightarrow f^{*}$ :

Theorem. Let $f, g \in L(X)$ and

$$
f^{*}=g^{*}
$$

almost everywhere. Then

$$
f(x)=g(x)
$$

for a.a. $x \in X$ (with respect to measure $\mu$ ).

A slightly weaker version of the theorem is formulated without proof in [3]. The analogous theorem in the discrete case is proved in [4].

2000 Mathematics Subject Classification: 28D10.

Servicio de Publicaciones. Universidad Complutense. Madrid, 2002 
Remark. Condition (1) is necessary for the validity of the theorem. If $\mu(X)=\infty$, then $f^{*}=0$ a.e. for every negative integrable $f$, since

$$
\lim _{t \rightarrow \infty} \frac{1}{t} \int_{0}^{t} f\left(T_{\tau} x\right) d \tau=0
$$

for a.a. $x \in X$ because of the Ergodic Theorem (see [1]).

First we need several lemmas.

Lemma 1. Let $f \in L(X)$. Then

$$
\operatorname{essinf} f^{*}=\frac{1}{\mu(X)} \int_{X} f d \mu \equiv \lambda_{0} .
$$

Proof. That $f^{*} \geq \lambda_{0}$ a.e. follows from the Ergodic Theorem:

$$
\lim _{t \rightarrow \infty} \frac{1}{t} \int_{0}^{t} f\left(T_{\tau} x\right) d \tau=\lambda_{0} \text { for a.a. } x \in X
$$

(see [1], [6]). The Maximal Ergodic Equality asserts that

$$
\mu\left(f^{*}>\lambda\right)=\frac{1}{\lambda} \int_{\left(f^{*}>\lambda\right)} f d \mu, \quad \lambda \geq \lambda_{0}
$$

(see [6], [2]), and if $\mu\left(f^{*}>\lambda\right)=\mu(X)$ for some $\lambda>\lambda_{0}$, we would get from (4) that $\mu(X)=\lambda^{-1} \int_{X} f d \mu$. This implies $\lambda=\lambda_{0}$, which is a contradiction.

Lemma 2. Let $\left(T_{t}\right)_{t \geq 0}$ be an ergodic semigroup of measure-preserving transformations on a finite measure space $(X, \mathbb{S}, \mu)$ and let $f \in L(X)$. Then

$$
f(x)=\lambda_{0} \quad \text { for a. } a . x \in\left(f^{*}=\lambda_{0}\right) .
$$

Proof. The Local Ergodic Theorem,

$$
\lim _{t \rightarrow 0+} \frac{1}{t} \int_{0}^{t} f\left(T_{\tau} x\right) d \tau=f(x)
$$

(see [6]), implies that

$$
f \leq \lambda_{0} \text { a.e. on }\left(f^{*}=\lambda_{0}\right) .
$$


On the other hand we have

$$
\lambda_{0} \mu(X)=\lambda_{0}\left(\mu\left(f^{*}>\lambda_{0}\right)+\mu\left(f^{*}=\lambda_{0}\right)\right)=\int_{\left(f^{*}>\lambda_{0}\right)} f d \mu+\int_{\left(f^{*}=\lambda_{0}\right)} f d \mu .
$$

Thus

$$
\lambda_{0} \mu\left(f^{*}=\lambda_{0}\right)=\int_{\left(f^{*}=\lambda_{0}\right)} f d \mu
$$

because of Maximal Ergodic Equality (see (4)). It follows from (6) and (7) that (5) holds.

For a locally integrable function $\xi$ on $\mathbb{R}_{0}^{+}=\{t \in \mathbb{R}: t \geq 0\}, \xi \in$ $L_{\text {loc }}\left(\mathbb{R}_{0}^{+}\right)$, the maximal operator $M$ is defined by

$$
M \xi(t)=\sup _{\tau>t} \frac{1}{\tau-t} \int_{t}^{\tau} \xi d m
$$

( $m$ is the Lebesgue measure on $\mathbb{R}$ ). Hence, if $\xi(t)=f\left(T_{t} x\right)$, then

$$
M \xi(t)=f^{*}\left(T_{t} x\right) .
$$

Obviously, for each $\lambda$ the set $(M \xi>\lambda)=\left\{t \in \mathbb{R}_{0}^{+}: M \xi(t)>\lambda\right\}$ is open (in $\mathbb{R}_{0}^{+}$). We shall use the following well-known facts about the connected components of this set (see [5], p.58):

If $\langle a, b), 0 \leq a<b<\infty$, (the sign $\langle$ before $a$ indicates that $a$ belongs or does not belong to the interval, i.e. $\langle a, b)=(a, b)$ or $\langle a, b)=[a, b))$ is a finite connected component of $(M \xi>\lambda)$, then

$$
\frac{1}{b-t} \int_{t}^{b} \xi d m>\lambda
$$

for each $t \in\langle a, b)$. If, in addition, $a \notin(M \xi>\lambda)$ i.e. $\langle a, b)=(a, b)$, then

$$
\frac{1}{b-a} \int_{a}^{b} \xi d m=\lambda
$$

Lemma 3. If $\xi, \eta \in L_{\mathrm{loc}}\left(\mathbb{R}_{0}^{+}\right)$and $M \xi=M \eta$ almost everywhere, then $M \xi(t)=M \eta(t)$ for all $t \geq 0$.

Proof. Let us show that for each $\xi \in L_{\text {loc }}\left(\mathbb{R}_{0}^{+}\right)$we have

$$
M \xi(t)=\lim _{\delta \rightarrow 0+} \operatorname{essinf}_{\tau \in(t, t+\delta)} M \xi(\tau), \quad t \geq 0,
$$


which obviously implies the validity of the lemma.

If $M \xi(t)>\lambda$, then there exists $\delta>0$ such that $M \xi(\tau)>\lambda$ for each $\tau \in(t, t+\delta)$. Thus

$$
M \xi(t) \leq \lim _{\delta \rightarrow 0+} \operatorname{essinf}_{\tau \in(t, t+\delta)} M \xi(\tau) .
$$

Conversely, if $M \xi>\lambda$ a.e. on $(t, t+\delta)$, then let us show that

$$
M \xi(t) \geq \lambda
$$

which finishes the proof.

Indeed, if $(t, t+\delta) \subset(M \xi>\lambda)$, then for each $\tau \in(t, t+\delta)$ we have $\sup \left\{\tau^{\prime}>\tau: \frac{1}{\tau^{\prime}-\tau} \int_{\tau}^{\tau^{\prime}} \xi d m \geq \lambda\right\} \geq t+\delta$ (see [5], p.58). Consequently, there exists $\tau^{\prime} \geq t+\delta$ such that

$$
\frac{1}{\tau^{\prime}-\tau} \int_{\tau}^{\tau^{\prime}} \xi d m \geq \lambda
$$

Set $\tau_{n} \searrow t$ and let

$$
\tau_{n}^{\prime}>t+\delta
$$

be such that

$$
\frac{1}{\tau_{n}^{\prime}-\tau_{n}} \int_{\tau_{n}}^{\tau_{n}^{\prime}} \xi d m \geq \lambda
$$

$n=1,2, \ldots$ Then

$$
\begin{gathered}
M \xi(t) \geq \frac{1}{\tau_{n}^{\prime}-t} \int_{t}^{\tau_{n}^{\prime}} \xi d m \geq \\
\left(\frac{1}{\tau_{n}^{\prime}-\tau_{n}} \int_{\tau_{n}}^{\tau_{n}^{\prime}} \xi d m-\frac{1}{\tau_{n}^{\prime}-\tau}\left|\int_{t}^{\tau_{n}} \xi d m\right|\right) \frac{\tau_{n}^{\prime}-\tau_{n}}{\tau_{n}^{\prime}-t}
\end{gathered}
$$

and taking into account that $\tau_{n} \rightarrow t, \tau_{n}^{\prime}-\tau \not \rightarrow 0$ (because of (12)) and $\left(\tau_{n}^{\prime}-\tau_{n}\right) /\left(\tau_{n}^{\prime}-t\right) \rightarrow 1$ as $n \rightarrow \infty$, we shall get (11).

If $\tau \notin(M \xi>\lambda)$ for some $\tau \in(t, t+\delta)$, then $(t, \tau)$ is covered up to a set of measure 0 with the connected components of $(M \xi>\lambda)$. In other words, there exist connected components $\Delta_{i}, i=1,2, \ldots$ such that $\Delta_{i} \subset(t, \tau)$ and $m\left((t, \tau) \backslash\left(\cup_{i=1} \Delta_{i}\right)\right)=0$. Since

$$
\frac{1}{m\left(\Delta_{i}\right)} \int_{\Delta_{i}} \xi d m=\lambda
$$


for each $i$ (see (10)), we have

$$
\int_{t}^{\tau} \xi d m=\lambda(\tau-t)
$$

and (11) holds.

The lemma below is actually proved in [3]. It is given here for the sake of completeness.

Lemma 4. Let $\xi \in L_{\mathrm{loc}}\left(\mathbb{R}_{0}^{+}\right)$, and let $\langle a, b)$ be a finite connected component of $(M \xi>\lambda)$ for some $\lambda$. Then the values $M \xi(t), t \in\langle a, b)$, uniquely define the values $\xi(t)$ for a.a. $t \in\langle a, b)$.

Hence, if another function $\eta \in L_{\mathrm{loc}}\left(\mathbb{R}_{0}^{+}\right)$is given such that $M \xi(t)=$ $M \eta(t), t \geq 0$, then $\xi(t)=\eta(t)$ for a.a. $t \in\langle a, b)$.

Proof. We shall show that the values $M \xi(t), t \in\langle a, b)$, uniquely define the function

$$
h(t)=\int_{t}^{b} \xi d m, \quad t \in\langle a, b) .
$$

Assume $t$ fixed and let $\lambda_{t}=M \xi(t)$. For each $\gamma \in\left[\lambda, \lambda_{t}\right)$ suppose $\left\langle a_{\gamma}, b_{\gamma}\right)$ to be the connected component of $(M \xi>\lambda)$ which contains $t$ and suppose $b_{\gamma}=t$ whenever $\gamma=\lambda_{t}$ (note that $b_{\lambda}=b$, by hypothesis). Obviously, $\left\langle a_{\gamma}, b_{\gamma}\right) \subset\left\langle a_{\gamma^{\prime}}, b_{\gamma^{\prime}}\right), \lambda_{t}>\gamma>\gamma^{\prime} \geq \lambda$, and

$$
\cup_{\gamma^{\prime}>\gamma}\left\langle a_{\gamma^{\prime}}, b_{\gamma^{\prime}}\right)=\left\langle a_{\gamma}, b_{\gamma}\right), \quad \lambda_{t}>\gamma \geq \lambda .
$$

It is easy to show that $\Psi: \gamma \rightarrow b_{\gamma}$ is a non-increasing function on $\left[\lambda, \lambda_{t}\right]$ continuous from the right. Observe also that $\Psi$ is uniquely defined by the values $M \xi(t), t \geq 0$.

Let $D$ be the set of points of discontinuity of this function, set

$$
b_{\gamma}^{\prime}=\lim _{\gamma^{\prime} \rightarrow \gamma-} b_{\gamma^{\prime}}
$$

for $\gamma \in D$, and let

$$
C=\left\{\gamma \in\left[\lambda, \lambda_{t}\right]: b_{\gamma^{\prime}}=b_{\gamma} \text { for some } \gamma^{\prime}>\gamma\right\} .
$$

Then the interval $[t, b]$, as a range of the non-increasing continuous from the right function $\Psi$, can be divided into pairwise disjoint parts:

$$
[t, b]=E_{1} \cup E_{2} \cup E_{3},
$$


where

$$
\begin{array}{r}
E_{1}=\left\{b_{\gamma}=\Psi(\gamma): \gamma \in\left[\lambda, \lambda_{t}\right] \backslash(D \cup C)\right\}, \\
E_{2}=\cup_{\gamma \in D}\left[b_{\gamma}, b_{\gamma}^{\prime}\right]
\end{array}
$$

and $\left.E_{3}=\left\{b_{\gamma}=\Psi(\gamma): \gamma \in C\right)\right\}$. Note that $E_{3}$ is a countable set and the intervals $\left(b_{\gamma}, b_{\gamma}^{\prime}\right)_{\gamma \in D}$ are disjoint.

Observe also that for each $e \in E_{1}$ there exists unique $\gamma \in\left[\lambda, \lambda_{t}\right]$ such that $e=b_{\gamma}=\Psi(\gamma)$. Hence, $\Psi^{-1}$ exists on $E_{1}$.

If $\gamma \in\left[\lambda, \lambda_{t}\right) \backslash(D \cup C)$ and $b_{\gamma} \in E_{1}$ is a Lebesgue point of $\xi$ then

$$
\xi\left(b_{\gamma}\right) \leq \gamma
$$

(since $M \xi\left(b_{\gamma}\right) \leq \gamma$ ). On the other hand, for each $\gamma^{\prime} \in\left(\gamma, \lambda_{t}\right)$ we have

$$
\frac{1}{b_{\gamma}-b_{\gamma^{\prime}}} \int_{b_{\gamma^{\prime}}}^{b_{\gamma}} \xi d m>\gamma
$$

since $\left\langle a_{\gamma}, b_{\gamma}\right)$ is a connected component of $(M \xi>\gamma)$ and $b_{\gamma^{\prime}} \in\left\langle a_{\gamma}, b_{\gamma}\right)$ (see (9)). Hence, taking into account that $b_{\gamma^{\prime}} \rightarrow b_{\gamma}$ when $\gamma^{\prime} \rightarrow \gamma$, we can conclude that $\xi\left(b_{\gamma}\right) \geq \gamma$, which together with (18) implies that

$$
\xi\left(b_{\gamma}\right)=\gamma .
$$

Thus $\xi=\Psi^{-1}$ a.e. on $E_{1}$ (see (16)) and consequently

$$
\int_{E_{1}} \xi d m=\int_{E_{1}} \Psi^{-1} d m
$$

If $\gamma \in D$, then

$$
\frac{1}{b_{\gamma}^{\prime}-b_{\gamma}} \int_{b_{\gamma}}^{b_{\gamma}^{\prime}} \xi d m \leq \gamma
$$

(since $M \xi\left(b_{\gamma}\right) \leq \gamma$ ) and for each $\gamma^{\prime} \in(\lambda, \gamma)$ we have

$$
\frac{1}{b_{\gamma^{\prime}}-b_{\gamma}} \int_{b_{\gamma}}^{b_{\gamma^{\prime}}} \xi d m>\gamma^{\prime}
$$

since $\left\langle a_{\gamma^{\prime}}, b_{\gamma^{\prime}}\right)$ is a connected component of $\left(M \xi>\gamma^{\prime}\right)$ and $b_{\gamma} \in\left\langle a_{\gamma^{\prime}}, b_{\gamma^{\prime}}\right)$ (see (9)). Hence, letting $\gamma^{\prime}$ converge to $\gamma$ from the left and taking into account (14), we get

$$
\frac{1}{b_{\gamma}^{\prime}-b_{\gamma}} \int_{b_{\gamma}}^{b_{\gamma}^{\prime}} \xi d m \geq \gamma
$$


This together with (20) implies that

$$
\int_{b_{\gamma}}^{b_{\gamma}^{\prime}} \xi d m=\gamma\left(b_{\gamma}^{\prime}-b_{\gamma}\right)
$$

Hence

$$
\int_{E_{2}} \xi d m=\sum_{\gamma \in D} \gamma\left(b_{\gamma}^{\prime}-b_{\gamma}\right)
$$

(see (17)). It follows from (13), (15), (19) and (21) that

$$
h(t)=\int_{E_{1}} \Psi^{-1} d m+\sum_{\gamma \in D} \gamma\left(b_{\gamma}^{\prime}-b_{\gamma}\right) .
$$

Thus $h(t)$ is uniquely defined by the function $\Psi$.

Corollary. Let $\xi, \eta \in L_{\mathrm{loc}}\left(\mathbb{R}_{0}^{+}\right)$be such that

$$
M \xi(t)=M \eta(t), \quad t \geq 0 .
$$

If $0 \leq t<t^{\prime}$ and

$$
M \xi(t)=M \eta(t)>M \xi\left(t^{\prime}\right)=M \eta\left(t^{\prime}\right),
$$

then

$$
\xi(\tau)=\eta(\tau)
$$

for a.a. $\tau$ from some neighbourhood of $t$.

Proof. If we take $\lambda \in\left(M \xi\left(t^{\prime}\right), M \xi(t)\right)$, then $t^{\prime} \notin(M \xi>\lambda)$ and some finite connected component of $(M \xi>\lambda)$ includes $t$. For a.a. $\tau$ from this interval (22) holds by virtue of the lemma.

Proof of Theorem. Equality (2) implies that

$$
\operatorname{ess} \inf f^{*}=\operatorname{essinf} g^{*} \equiv \lambda_{0} .
$$

Consequently,

$$
\mu\left(f^{*}<\lambda\right)=\mu\left(g^{*}<\lambda\right)>0 \text { for all } \lambda>\lambda_{0}
$$

and

$$
\mu\left(f^{*}<\lambda_{0}\right)=\mu\left(g^{*}<\lambda_{0}\right)=0
$$


Define

$$
\xi_{x}(t)=f\left(T_{t} x\right) \quad \text { and } \quad \eta_{x}(t)=g\left(T_{t} x\right), \quad x \in X, t \geq 0 .
$$

We shall prove that for a.a. $x \in X$

$$
m\left\{t \geq 0: \xi_{x}(t) \neq \eta_{x}(t)\right\}=0 .
$$

Obviously, this implies that

$$
\mu(f \neq g)=0 .
$$

(If $X_{1} \subset X$ and $\mu\left(X_{1}\right)>0$ then, by the Ergodic Theorem, see (3),

$$
m\left\{t \geq 0: T_{t} x \in X_{1}\right\}=\lim _{t \rightarrow \infty} \int_{0}^{t} \mathbb{I}_{X_{1}}\left(T_{\tau} x\right) d \tau=\infty
$$

for a.a. $x \in X$, while

$$
\left.\left\{t \geq 0: \xi_{x}(t) \neq \eta_{x}(t)\right\}=\left\{t \geq 0: T_{t} x \in(f \neq g)\right\}, \quad x \in X .\right)
$$

If $X_{0} \subset X$ and $\mu\left(X_{0}\right)=0$, then by standard application of Fubini's theorem we have

$$
m\left\{t \geq 0: T_{t} x \in X_{0}\right\}=0
$$

for a.a. $x \in X$. Hence

$$
m\left\{t \geq 0: M \xi_{x}(t) \neq M \eta_{x}(t)\right\}=m\left\{t \geq 0: T_{t} x \in\left(f^{*} \neq g^{*}\right)\right\}=0
$$

for a.a. $x \in X$ (see (2), (8)) and Lemma 3 implies that

$$
M \xi_{x}(t)=M \eta_{x}(t), \quad t \geq 0,
$$

for a.a. $x \in X$. We also have

$$
m\left\{t \geq 0: M \xi_{x}(t)=M \eta_{x}(t)<\lambda_{0}\right\}=0
$$

(see (24)) and

$$
m\left\{t \geq 0: M \xi_{x}(t)=M \eta_{x}(t)=\lambda_{0}, \quad \xi_{x}(t) \neq \lambda_{0} \text { or } \eta_{x}(t) \neq \lambda_{0}\right\}=0
$$

for a.a. $x \in X$ (see (5)).

We consider two cases: 
(i) $\mu\left(f^{*}=\lambda_{0}\right)=\mu\left(g^{*}=\lambda_{0}\right)>0$. Then

$$
m\left\{t \geq 0: M \xi_{x}(t)=M \eta_{x}(t)=\lambda_{0}\right\}=\infty
$$

for a.a. $x \in X$ (see (26)). Take $x \in X$ for which (28), (29), (30) and (31) hold (note that almost all $x$ have this property). Let $E=\{t \geq 0$ : $\left.M \xi_{x}(t)=M \eta_{x}(t)>\lambda_{0}\right\}$. Then for each $t \in E$ there exists $t^{\prime}>t$ such that $M \xi_{x}\left(t^{\prime}\right)=M \eta_{x}\left(t^{\prime}\right)=\lambda_{0}$, because of (31). Thus the corollary of Lemma 4 implies that

$$
\xi_{x}(t)=\eta_{x}(t)
$$

for a.a. $t \in E$.

It follows from (29) and (30) that $\xi_{x}(t)=\eta_{x}(t)=\lambda_{0}$ for a.a. $t \in$ $\mathbb{R}_{0}^{+} \backslash E$. Thus (32) holds for a.a. $t \geq 0$ and (25) is valid.

(ii) $\mu\left(f^{*}=\lambda_{0}\right)=\mu\left(g^{*}=\lambda_{0}\right)=0$. Then

$$
m\left\{t \geq 0: M \xi_{x}(t)=M \eta_{x}(t) \leq \lambda_{0}\right\}=0
$$

for a.a. $x \in X$ (see (8), (24) and (27))

If $\lambda_{i}$ is any decreasing sequence convergent to $\lambda_{0}, \lambda_{i} \searrow \lambda_{0}$, then

$$
\mu\left(f^{*}<\lambda_{i}\right)=\mu\left(g^{*}<\lambda_{i}\right)>0, \quad i=1,2, \ldots
$$

(see (23)) and consequently for a.a. $x \in X$ we have

$$
\begin{gathered}
m\left\{t \geq 0: M \xi_{x}(t)=M \eta_{x}(t)<\lambda_{i}\right\}= \\
m\left\{t \geq 0: f^{*}\left(T_{t} x\right)=g^{*}\left(T_{t} x\right)<\lambda_{i}\right\}=\infty, \quad i=1,2, \ldots,
\end{gathered}
$$

(see (26)). Take $x \in X$ for which (28), (33) and (34) hold (note that almost all $x$ have this property). It follows from (33) and (34) that for a.a. $t \geq 0$ there exists $t^{\prime}>t$ such that

$$
M \xi_{x}(t)=M \eta_{x}(t)>M \xi_{x}\left(t^{\prime}\right)=M \eta_{x}\left(t^{\prime}\right) .
$$

Thus, by virtue of the corollary of Lemma 4, (32) holds for a.a. $t \geq 0$ and $(25)$ is valid.

\section{References}

[1] I.P. Cornfeld, S.V. Fomin and Ya.G. Sinai, Ergodic Theory, Springer-Verlag, Berlin, 1983. 
[2] L. Ephremidze, On the majorant of ergodic means (continous case) (Russian), Trudy Tbiliss. Mat. Inst. Razmadze Akad. Nauk Gruz. SSR, 98 (1990), 112-124.

[3] _ On the uniqueness of maximal functions, Georgian Math. J., 3 (1996), 49-52.

[4] On the uniqueness of the ergodic maximal function, (to appear).

[5] M. Guzmán, Differentiation of integrals in $R^{n}$, Springer-Verlag, Berlin, 1975 .

[6] K.E. Petersen, Ergodic Theory, Cambridge Univ. Press, Cambridge, 1983.

A. Razmadze Mathematical Institute

Aleksidze 1

380093 Tbilisi

Georgia

E-mail: lasha@rmi.acnet.ge

Current address: Mathematical Institute

Zitna 25, 11567

Praha 1

Czech Republic

E-mail: lasha@math.cas.cz

Recibido: 23 de Enero de 2001

Revisado: 7 de Junio de 2001 